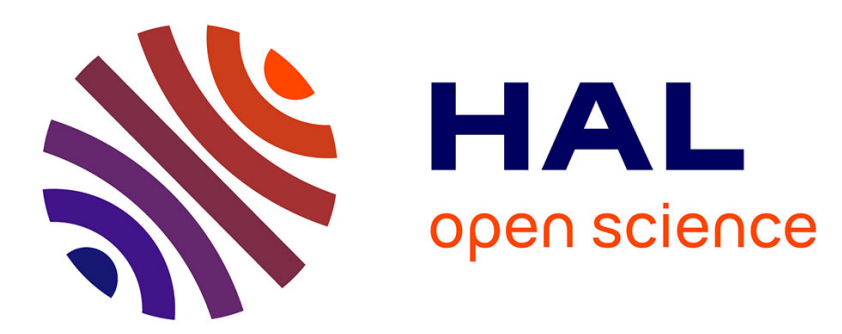

\title{
Channel Shortening for OFDM Systems: An Improved Algorithm in Noisy Environments
}

Papa Moussa Ndao, Maryline Hélard, Matthieu Crussière, François Yven

\section{To cite this version:}

Papa Moussa Ndao, Maryline Hélard, Matthieu Crussière, François Yven. Channel Shortening for OFDM Systems: An Improved Algorithm in Noisy Environments. ICUWB 2014, Sep 2014, Paris, France. hal-01113797

\author{
HAL Id: hal-01113797 \\ https://hal.science/hal-01113797
}

Submitted on 6 Feb 2015

HAL is a multi-disciplinary open access archive for the deposit and dissemination of scientific research documents, whether they are published or not. The documents may come from teaching and research institutions in France or abroad, or from public or private research centers.
L'archive ouverte pluridisciplinaire HAL, est destinée au dépôt et à la diffusion de documents scientifiques de niveau recherche, publiés ou non, émanant des établissements d'enseignement et de recherche français ou étrangers, des laboratoires publics ou privés. 


\title{
Channel Shortening for OFDM Systems: An Improved Algorithm in Noisy Environments
}

\author{
Papa Moussa Ndao, Maryline Hélard, Matthieu Crussière, François Yven \\ Institut d'Electronique et des Télécommunications de Rennes (IETR), UMR 6164 \\ INSA Rennes, France \\ \{papa-moussa.ndao, maryline.helard, matthieu.crussiere, francois.yven \} @ insa-rennes.fr
}

\begin{abstract}
In this paper, we propose an efficient channel shortening algorithm, applied to OFDM systems, exploiting a particular decomposition of the Toeplitz convolution channel matrix and of the channel shortening filter (CSF). Unlike classical methods which optimize the CSF following one single criterion applied to the whole response of the filter, our decomposition allows for addition of complementary criteria. Similarly to classical CS techniques, the proposed method tries to concentrate most of the energy of the shortened channel impulse response (SCIR) within the tolerated delay-spread window. However, our second applied criterion aims at limiting the noise enhancement on each sub-carrier by minimizing the spectral distortions related to the filtering function. The performance of the algorithm in terms of computational complexity and bit error rate (BER) is studied by simulations and compared to the reference algorithm of the literature referred to as maximum shortening signal to noise ratio (MSSNR) algorithm.
\end{abstract}

Index Terms - OFDM, channel shortening, spectral distortion minimization.

\section{INTRODUCTION}

$I^{2}$ $\mathrm{N}$ multicarrier communication systems such as orthogonal frequency division multiplexing (OFDM), a guard interval is usually inserted between symbols to mitigate inter-symbol interference (ISI). When the channel delay spread is larger than the guard interval length, performance can drastically degrade. This phenomenon can be encountered in UWB communications as for example for some severe NLOS channels and high rate transmissions for which error floor appears [1]. Channel shortening (CS) technique was demonstrated to be an efficient technique to provide a smaller equivalent channel length to the OFDM demodulator [2]. CS can also be applied to allow the use of a shorter guard interval, thus leading to a better spectral efficiency. Then, it can be used in UWB systems, where channels are known to be very rich in multipaths.

Various CS techniques applied to OFDM systems have already been proposed and discussed, however always considering that the CSF coefficients should be obtained through a one-shot computation, either by maximizing a given criterion or by minimizing the channel delay spread [3][2][4]. These classical algorithms have good BER performance, but lead to high computation complexity.

One of the most famous and efficient CS techniques is the so-called maximum shortening signal to noise ratio (MSSNR) algorithm [5], that defines the CSF on the basis of a global channel energy optimization with a classical linear filter. The goal of this algorithm is to find the CSF that minimizes the energy of the shortened channel impulse response (SCIR) outside a target window, while keeping the amount of energy as constant inside that window. Another technique proposed in [6] is based on energy optimization but assumes CSF decomposition into two parts. In this method, the first part of the filter is dedicated to concentrate the SCIR energy in the desired window, while the second part of the filter is used to cancel the energy outside. Both of these techniques can however suffer from noise power boost in some situations where the channel shortening processing translates into the accentuation of some deep fades of the channel frequency response.

In this paper, we propose an efficient CS method relying on a two-part CSF as in [6] but redesigning the two optimization criteria in a complementary fashion so that the noise power boost effect mentioned before is mitigated. The first part of the CSF is used to concentrate all energy in a small desired window. The second part of the filter is exploited to satisfy the second criterion that is chosen to limit the spectral distortion between the original channel response and the shortened one, hereby avoiding strong noise power increase after CS filtering. The proposed 2-part CS provides a lower complexity solution and a good bit error rate (BER) performance, compared with the classical 1-part CS MSSNR.

The remainder of this paper is organized as follows. Section II presents the channel shortening principle and section III the system model. Section IV is dedicated to the design of the proposed method. Section $\mathrm{V}$ gives comparative performance results. Finally, VI concludes the paper.

\section{Notations}

1) Boldface lower case letters represent vectors, and boldface upper case letters are reserved for matrices. The notation $\boldsymbol{A}^{H}$ denotes transpose-conjugate of $\boldsymbol{A}$ and $\boldsymbol{A}^{T}$ the transpose of $\boldsymbol{A}$.

2) Non-boldfaced upper case letters are reserved for matrix frequency domain representation.

3) The notation $F$ is used to represent the $N \times N$ unitary DFT matrix given by 


$$
F_{i j}=\frac{1}{\sqrt{N}} e^{-j(2 \pi / N) i j}, \quad \text { for } \quad 0 \leq i, j \leq N-1
$$

\section{CHANNEL SHORTENING PRINCIPLE}

\section{A. Generic system}

We consider a baseband OFDM system (as shown in Fig. 1). In this model, samples $x(k)$ from the source are grouped into blocks of size $N$ equals to the number of subcarriers. Modulation and demodulation are performed by using simple inverse fast Fourier transform (IFFT) and FFT operations. The channel is assumed to be estimated at the receiver.

Channel shortening is performed by a filter $\boldsymbol{w}$ placed at the receiver (Fig. 1). Then, the received signal $y(k)$ is the transmitted signal $x(k)$ affected by the shortened channel impulse response (SCIR) designed by $\tilde{\boldsymbol{h}}$ and the noise sample $n(k)$.

The SCIR represents the combined effect of the channel impulse response (CIR) $\boldsymbol{h}$ and the CSF $\boldsymbol{w}$.

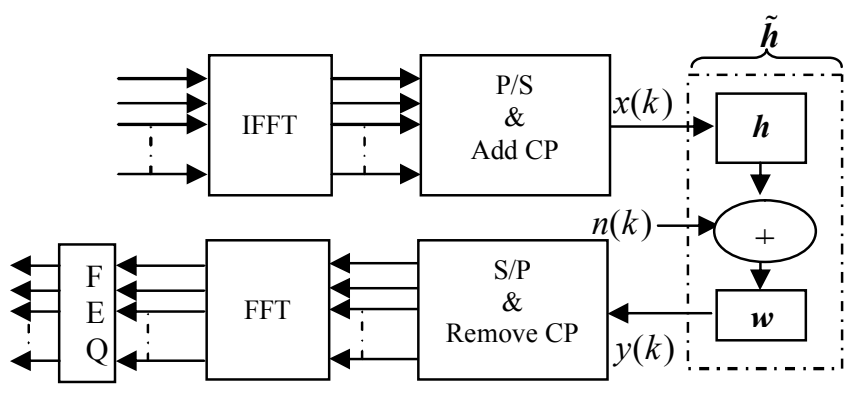

Fig. 1. Communication system with CSF at the receiver.

\section{B. Synchronization and channel estimation}

In OFDM systems, a time synchronization of data blocks is necessary at the receiver to detect the symbol start. A classical solution for this is to process a sliding window to detect autocorrelation peaks in the received signal due to the presence of the cyclic prefix [7].

After this synchronization step, a least-squares algorithm based on pilot tones is generally used to estimate the channel in the frequency domain. It is then possible to estimate the CIR and to track the first significant path in the estimated CIR to refine time synchronization. This estimated and finelysynchronized CIR can further be used by any CS algorithm, e.g. MSSNR, to compute the CSF coefficients.

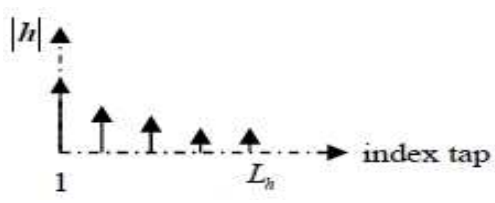

Fig. 2. Illustration of the CIR estimated after synchronization on the first path

\section{Channel shortening algorithm, re-synchronization and equalization of MSSNR [5]}

The delay spread of the SCIR is contained in a reduced window of size $L_{\max }<L_{h}$, but a shift $d$ can occur depending on the way the CS is performed.

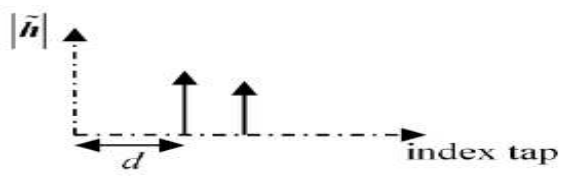

Fig. 3. Illustration of the SCIR estimated

In classical methods such as MSSNR, a window is placed on different positions by considering various possibilities of delays values. The objective is to search the ideal position that gives the optimal CSF. Then, if the algorithm finds the best CSF for a particular delay $d$, a new and simple synchronization operation is necessary before the equalization step. To do this, the first $d$ samples of the received signal $y$ have to be dropped, as well as the first $d$ samples of the shortened channel $\tilde{\boldsymbol{h}}$. The design of the MSSNR CSF, for a particular delay $d$ is described in appendix VII.

At last, the OFDM equalization can be performed by using a frequency domain equalizer (FEQ), such as the zero forcing (ZF) equalizer that is based on the inversion of the gain of the synchronized shortened channel, estimated for each subcarrier.

\section{SYSTEM MODELS}

\section{A. Shortening system in one shot}

Using a column vector representation, we define the CIR $\boldsymbol{h}$ of size $L_{h}$ and the CSF $\boldsymbol{w}$ of size $L_{w}$ as follows

$$
\begin{gathered}
\boldsymbol{h}=\left[h_{0}, h_{1} \cdots, h_{L_{h}-1}\right]^{T} \\
\boldsymbol{w}=\left[w_{0}, \cdots, w_{L_{w}-2}, w_{L_{w}-1}\right]^{T}
\end{gathered}
$$

The convolution between these two impulse responses yields the following column vector

$$
\tilde{\boldsymbol{h}}=\boldsymbol{h} \otimes \boldsymbol{w}=\left[\tilde{h}_{0}, \cdots, \tilde{h}_{\tilde{L}-2}, \tilde{h}_{\tilde{L}-1}\right]^{T}
$$

of length $\tilde{L}=L_{h}+L_{w}-1$, where $\otimes$ is the convolution operator. Defining appropriate notations, (4) can be written as

$$
\tilde{\boldsymbol{h}}=\boldsymbol{H} \boldsymbol{w}
$$

where $\boldsymbol{H}$ is the Toeplitz convolution matrix of size $\tilde{L} \times L_{w}$ and defined as 


$$
\boldsymbol{H}=\left[\begin{array}{lllll}
h_{0} & 0 & \cdots & \ldots & 0 \\
h_{1} & h_{0} & \ddots & & \vdots \\
\vdots & \vdots & & & \\
h_{L_{h}-1} & h_{L_{h}-2} & \cdots & h_{L_{h}-L_{w}+1} & h_{L_{h}-L_{w}} \\
0 & h_{L_{h}-1} & \cdots & & h_{L_{h}-L_{w}+1} \\
\vdots & \ddots & & & \\
0 & \cdots & & 0 & h_{L_{h}-1}
\end{array}\right]
$$

\section{B. Two-part channel shortening filter (2P-CSF)}

We use the CSF decomposition adopted in [6]. Then, $\boldsymbol{w}$ is divided into two parts as

$$
\boldsymbol{w}^{T}=\left[\begin{array}{ll}
\boldsymbol{w}_{\max }^{T} & \boldsymbol{w}_{\min }^{T}
\end{array}\right]
$$

where $\boldsymbol{w}_{\text {max }}=\left[w_{0}, \cdots, w_{L_{\max }}\right]^{T}$ and $\boldsymbol{w}_{\text {min }}=\left[w_{L_{\max }+1}, \cdots, w_{L_{w}-1}\right]^{T}$. Then, relation (5) can be represented as

$$
\tilde{\boldsymbol{h}}=\left[\begin{array}{ll}
\boldsymbol{H}_{1} & \boldsymbol{O} \\
\boldsymbol{H}_{2} & \boldsymbol{H}_{3}
\end{array}\right]\left[\begin{array}{l}
\boldsymbol{w}_{\max } \\
\boldsymbol{w}_{\min }
\end{array}\right]
$$

where $\boldsymbol{H}_{1}, \boldsymbol{H}_{2}$ and $\boldsymbol{H}_{3}$ are Toeplitz matrices, of size $L_{\max } \times L_{\max }, \tilde{L}-L_{\max } \times L_{\max }$ and $\tilde{L}-L_{\max } \times L_{w}-L_{\max }$ respectiveely. As for the 2P-CSF, the SCIR may be decomposed into two regions as follows

$$
\tilde{\boldsymbol{h}}=\left[\begin{array}{c}
\tilde{\boldsymbol{h}}_{\max } \\
\tilde{\boldsymbol{h}}_{\min }
\end{array}\right]=\left[\begin{array}{c}
\boldsymbol{H}_{1} \boldsymbol{w}_{\max } \\
\boldsymbol{H}_{2} \boldsymbol{w}_{\max }+\boldsymbol{H}_{3} \boldsymbol{w}_{\min }
\end{array}\right]
$$

Then, by equivalence, $\tilde{\boldsymbol{h}}_{\max }=\boldsymbol{H}_{1} \boldsymbol{w}_{\max }$ represents the desired window where the energy should be dominant and $\tilde{\boldsymbol{h}}_{\text {min }}=\boldsymbol{H}_{2} \boldsymbol{w}_{\text {max }}+\boldsymbol{H}_{3} \boldsymbol{w}_{\text {min }}$ the residual region, in which energy should be less pronounced.

\section{Channel representation in frequency domain for $2 P-C S F$}

Let $H$ and $\tilde{H}$ be the frequency domain representation of the CIR and the SCIR, respectively. After a zero-padded operation to get vectors columns of sizes $N$, we can write

$$
H=F\left[\begin{array}{c}
\boldsymbol{h} \\
\boldsymbol{0}_{\left(N-L_{h}\right)}
\end{array}\right] \text { and } \tilde{H}=F\left[\begin{array}{c}
\tilde{\boldsymbol{h}} \\
\boldsymbol{0}_{(N-\tilde{L})}
\end{array}\right]
$$

Substituting $\tilde{\boldsymbol{h}}$ by his expression given in (5), $\tilde{H}$ takes the following form

$$
\tilde{H}=F\left[\begin{array}{c}
\tilde{\boldsymbol{h}} \\
\boldsymbol{0}_{(N-\tilde{L})}
\end{array}\right]=\underbrace{F\left[\begin{array}{c}
\boldsymbol{H} \\
\boldsymbol{\boldsymbol { 0 }}_{\left(N-\tilde{L}, L_{w}\right)}
\end{array}\right]}_{\boldsymbol{P}} \boldsymbol{w}=\boldsymbol{P} \boldsymbol{w}
$$

where $\boldsymbol{P}$ is a $N \times L_{w}$ matrix. As the CSF and the SCIR, the matrix $\boldsymbol{P}$ is divided into 2 parts as follows

$$
\boldsymbol{P}=\left[\begin{array}{ll}
\boldsymbol{P}_{\max } & \boldsymbol{P}_{\min }
\end{array}\right]
$$

where $\boldsymbol{P}_{\max }$ and $\boldsymbol{P}_{\min }$, represents matrices constructed by considering the first $L_{\max }$ columns and the last columns of $\boldsymbol{P}$, respectively. Then, integrating (11) in (10) yields

$$
\tilde{H}=\boldsymbol{P}_{\max } \boldsymbol{w}_{\max }+\boldsymbol{P}_{\min } \boldsymbol{w}_{\min }
$$

We define a real-value function of complex vector variable, given for a fixed $\boldsymbol{w}_{\max }$ as

$$
\begin{aligned}
f_{\boldsymbol{w}_{\max }}: \mathbb{C}^{L_{\min }} & \rightarrow \mathbb{R} \\
\boldsymbol{w}_{\text {min }} & \rightarrow\|\tilde{H}\|_{2}^{2}=\boldsymbol{w}_{\max }^{H} \boldsymbol{A} \boldsymbol{w}_{\text {max }}+\boldsymbol{w}_{\max }^{H} \boldsymbol{B} \boldsymbol{w}_{\min }+ \\
& \boldsymbol{w}_{\min }^{H} \boldsymbol{C} \boldsymbol{w}_{\text {max }}+\boldsymbol{w}_{\min }^{H} \boldsymbol{D} \boldsymbol{w}_{\text {min }}
\end{aligned}
$$

where $\boldsymbol{A}=\boldsymbol{P}_{\max }^{H} \boldsymbol{P}_{\max }, \boldsymbol{B}=\boldsymbol{P}_{\max }^{H} \boldsymbol{P}_{\min }, \boldsymbol{C}=\boldsymbol{P}_{\min }^{H} \boldsymbol{P}_{\max }$, and $\boldsymbol{D}=\boldsymbol{P}_{\min }^{H} \boldsymbol{P}_{\min }$ are matrices of size $L_{\max } \times L_{\max }$,

$L_{\max } \times\left(L_{w}-L_{\max }\right),\left(L_{w}-L_{\max }\right) \times L_{\max }$ and $\left(L_{w}-L_{\max }\right) \times\left(L_{w}-L_{\max }\right)$, respectively. $\|_{2}$ is the 2-norm. $f_{w_{\text {max }}}$ will be used as a cost function in IV, to design the CSF.

\section{PROPOSED ALGORITHM}

\section{A. Calculation of the CSF coefficients}

Most of classical channel shortening methods aim at optimizing a performance criterion (SNR, SINR...). For instance the MSSNR technique aims at maximizing energy in the target window while minimizing it outside this window (see appendix). In this paper, thanks to the use of the 2P-CSF, we propose to apply two criterions: i) to maximize the energy into the target window and ii) to minimize spectral distortion to avoid noise increase when applying the CSF filter.

Our algorithm is made of 2 steps:

- The first step consists in computing $\boldsymbol{w}_{\min }$ as a function of $\boldsymbol{w}_{\max }$ in order to reduce the spectral distortions. We can then express $\tilde{\boldsymbol{h}}_{\text {min }}$ as a function of $\boldsymbol{w}_{\max }$.

- $\quad$ The second one consists in computing $\boldsymbol{w}_{\max }$ in order to concentrate all the energy of the SCIR within the allowed window and to minimize the energy outside thanks to the Rayleigh quotient.

Step 1: the second part of the CSF, denoted by $\boldsymbol{w}_{\text {min }_{\text {opt }}}$ is given by the following optimization problem

$$
\boldsymbol{w}_{\text {min }_{\text {opt }}}=\underbrace{\arg \min }_{\boldsymbol{w}_{\text {min }}}\left\{\|\tilde{H}\|_{2}^{2}-\|H\|_{2}^{2}\right\}=\underbrace{\arg \min }_{\boldsymbol{w}_{\min }}\left\{\|\tilde{H}\|_{2}^{2}\right\}
$$

since $\|H\|_{2}^{2}$ is a constant for a given CIR. Then, solving (14) is equivalent to find the optimal solution $\boldsymbol{w}_{\text {min }_{\text {opt }}}$ that minimizes the function $f_{\boldsymbol{w}_{\max }}$ for a given $\boldsymbol{w}_{\max }$.

We define $\nabla_{z}$, the complex gradient operator with respect to the complex vector $z$ by

$$
\nabla_{z}=\left[\partial / \partial z_{1}, \partial / \partial z_{2}, \cdots, \partial / \partial z_{N}\right]^{T}
$$


Only one of the following conditions is necessary and sufficient to determine a stationary point of $f_{w_{\max }}$ [8].

$$
\nabla_{\boldsymbol{w}_{\min }^{H}} f_{\boldsymbol{w}_{\max }}\left(\boldsymbol{w}_{\min }\right)=0 \quad \text { or } \quad \nabla_{\boldsymbol{w}_{\min }} f_{\boldsymbol{w}_{\max }}\left(\boldsymbol{w}_{\min }\right)=0
$$

Thus, the optimal solution is given by

$$
\boldsymbol{w}_{\min _{\text {opt }}}=\underbrace{-\boldsymbol{D}^{-1} \boldsymbol{C}}_{\boldsymbol{K}_{1}} \boldsymbol{w}_{\max }=\boldsymbol{K}_{1} \boldsymbol{w}_{\max }
$$

Step2: We calculate now $\boldsymbol{w}_{\max }$ which allows for concentration of most of the energy of the SCIR inside the predefined target region and that simultaneously minimizes energy outside this window. Substituting (17) into (8), we obtain the new expression of $\tilde{\boldsymbol{h}}_{\text {min }}$ :

$$
\tilde{\boldsymbol{h}}_{\min }=\left(\boldsymbol{H}_{2}+\boldsymbol{H}_{3} \boldsymbol{K}_{1}\right) \boldsymbol{w}_{\max }
$$

Then, energy inside and outside the target window is given, respectively, by

$$
\begin{aligned}
\xi_{\text {max }} & =\tilde{\boldsymbol{h}}_{\max }^{H} \tilde{\boldsymbol{h}}_{\text {max }}=\boldsymbol{w}_{\text {max }}^{H} \boldsymbol{Q}_{\max } \boldsymbol{w}_{\text {max }} \\
\xi_{\text {min }} & =\tilde{\boldsymbol{h}}_{\min }^{H} \tilde{\boldsymbol{h}}_{\text {min }}=\boldsymbol{w}_{\text {max }}^{H} \boldsymbol{Q}_{\text {min }} \boldsymbol{w}_{\text {max }}
\end{aligned}
$$

where $\boldsymbol{Q}_{\max }=\boldsymbol{H}_{1}^{H} \boldsymbol{H}_{1}$ and $\boldsymbol{Q}_{\min }=\left(\boldsymbol{H}_{2}+\boldsymbol{H}_{3} \boldsymbol{K}_{1}\right)^{H}\left(\boldsymbol{H}_{2}+\boldsymbol{H}_{3} \boldsymbol{K}_{1}\right)$ are matrices of dimensions $L_{\max } \times L_{\max }$. Then, the optimal value of $\boldsymbol{w}_{\max }$ designed by $\boldsymbol{w}_{\text {max }_{\text {opt }}}$, is the solution of the following Rayleigh quotient problem (21).

$$
\boldsymbol{w}_{\text {max }_{\text {opt }}}=\underset{\boldsymbol{w}_{\max }}{\operatorname{Argmax}}\left\{\frac{\boldsymbol{w}_{\max }^{H} \boldsymbol{Q}_{\max } \boldsymbol{w}_{\max }}{\boldsymbol{w}_{\max }^{H} \boldsymbol{Q}_{\min } \boldsymbol{w}_{\max }}\right\}
$$

Finally, the optimal CSF is given by the concatenation of the two column vectors, $\boldsymbol{w}_{\text {max }_{\text {opt }}}$ and $\boldsymbol{w}_{\text {min }_{\text {opt }}}$.

\section{B. Computational complexity}

To figure out the computational complexity of the proposed algorithm, we count the number of complex multiplications required in the algorithm. The calculation for each method is done in two steps:

- In the first step, we compute $\boldsymbol{Q}_{\max }$ and $\boldsymbol{Q}_{\min }$ for the 2PCSF. For the MSSNR algorithm, matrices $\boldsymbol{A}_{d}$ and $\boldsymbol{B}_{d}$ (see appendix VII) are calculated for each delay.

- In the second step, we use the Rayleigh quotient problem in each method for which computation complexity is provided in [6] [8]. The number of multiplications needed for the 2PCSF is in the order of $11 L_{\max }^{3} / 3$ where $L_{\max }$ is the desired window size and is also the size of matrices $\boldsymbol{Q}_{\max }$ and $\boldsymbol{Q}_{\min }$. Note that it is also possible to control the complexity of our method by reducing $L_{\max }$. The MSSNR method requires a complexity of $11 L_{w}^{3} / 3$ for each delay $d$ where $L_{w}$ is the size of the CSF and is also equals to size of matrices $\boldsymbol{A}_{d}$ and $\boldsymbol{B}_{d}$. Over $N_{d}$ delay possible candidates, the number of multiplications is then approximately of order $\left(11 L_{w}^{3} / 3\right) N_{d}$. This leads to high computation complexity for a large value of $L_{w}$.
TABLE I

COMPLEXITY OF CHANNEL SHORTENING SCHEMES

\begin{tabular}{|c|c|}
\hline Algorithm & Total Complexity \\
\hline 2P-CSF & $\begin{array}{r}L_{\text {max }}^{3}(11 / 3+2)+\left(L_{w}-L_{\max }\right)^{3}+L_{w}^{2}\left(L_{\max }+N\right) \\
\end{array}$ \\
\hline MSSNR [10] & $\left\{L_{w}\left(N^{2}+L_{\max } \tilde{L}-3 L_{\max }^{2}-N L_{\max }\right)\right.$ \\
\hline
\end{tabular}

\section{Delay analysis}

It is shown that CSF is sensitive to delay parameter, when small size filter is used [10].

In our proposed method however, all energy of the SCIR is concentrated in the first coefficients. This is due to the particular decomposition of the CSF [6] in which the first part of the filter fixes the region where the energy should be dominant. Then, in the proposed method there is no need to include a delay search algorithm like MSSNR case (as shown in Fig. 3), to find the best CSF. But the second criterion that is chosen to avoid the strong noise power increase after CS filtering yields an optimal channel shortening filter with good BER performance.

On the other hand, the proposed algorithm is used for long channels delay spread, and imposes filter sizes equal or longer than the CIR size. Thus, performances sensitivity to delay parameter for small filter sizes can be ignored.

\section{SIMULATION RESULTS}

The algorithm to be compared with the proposed one is the classical MSSNR [5]. We also compare BER results with and without the use of the CSF. For all simulations, we assume perfect estimated channels and perfect synchronization on the first significant path of the CIR. For each algorithm, we take the optimal $L_{\max }$ that gives the best BER. Two examples of UWB channel models (CM) denoted CM4 and based on Saleh-Valenzuela for indoor applications [11], with approximately 63 significant taps $\left(L_{h}=62\right)$ and with different spectra, are used for simulations. For the rest of the article, we denote by $\mathrm{C} 1$ and $\mathrm{C} 2$ the 2 chosen channels over CM4 models.

These channels have delay spread longer than the longest guard interval specified for UWB transmissions with OFDM waveform leading to the well-known error floor phenomenon in BER performance. The noise is considered to be additive white Gaussian (AWGN). The FFT size, the filter size, and the size of the CP (cyclic prefix) used to mitigate inter-symbol interference, is $N=128, L_{w}=63$ and $v=33$, respectively. Uncoded 4-QAM and 16-QAM modulations are used.

Fig. 4 and 7 provide channel impulse response before and after channel shortening, for $\mathrm{C} 1$ and $\mathrm{C} 2$ respectively. Fig. 5 and 8 present the SNR for each subcarrier before and after channel shortening for $\mathrm{C} 1$ and $\mathrm{C} 2$ respectively that allow for representing the spectral distortion brought to the initial spectrum after CS. Fig. 6 and 9 provide BER performance of 
systems (without/with channel shortening).

Channel 1: with $L_{\max }=30$ for the two algorithms (MSSNR and proposed method). Delay optimization has been applied for the MSSNR technique to get the best CSF leading to $d=5$.

In Fig. 4, the shortened CIR is compared with the original one. Since CIR has significant energy outside the guard interval $(v=33)$, ISI leads to error floor phenomenon as observed on Fig. 6. As expected, with CS, energy is well concentrated inside the desired window. SNR observed after CS is represented on Fig. 5 are quite similar for both methods. As results, in Fig. 6, the BER of each system similarly improved and the error floor even disappears thanks to CS.

Complexity: in this case, the MSSNR algorithm requires approximately 132.445 .530 multiplications, where the proposed algorithm requires 1.670 .571 .

Channel 2: with $L_{\max }=28$ for the proposed method and 29 for the MSSNR. Delay optimization has been applied for the MSSNR technique to get the best CSF. Then $d=34$.

Here, the proposed method outperforms the MSSNR algorithm. In fact, our method does not only concentrate the energy inside the allowed window (Fig. 7), but also reduce the spectral distortion between the original and the resulting spectrum after CS as can be observed on Fig. 8. The deeper the fadings after $\mathrm{CS}$, the greater the noise power on the concerned subcarriers and thus the smaller the global performance (Fig. 9).

Complexity: in this case, the MSSNR algorithm requires approximately 133.854 .525 multiplications, where the proposed algorithm requires 1.663.400.
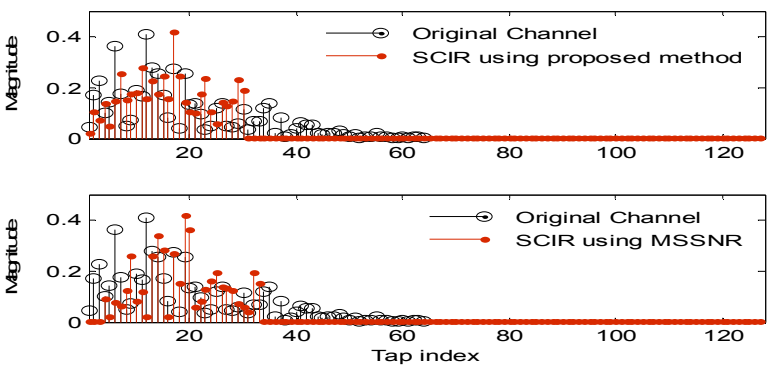

Fig. 4. The original CIR versus the normalized shortened channel C1

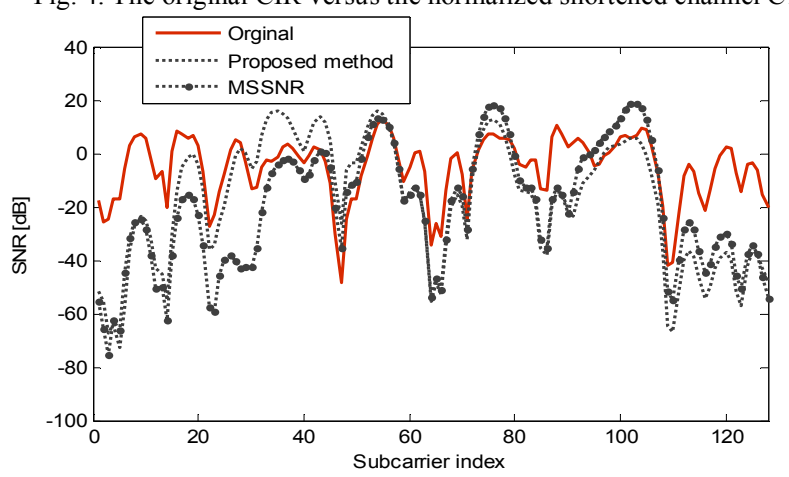

Fig. 5. SNR distribution over frequency for $\mathrm{C} 1$

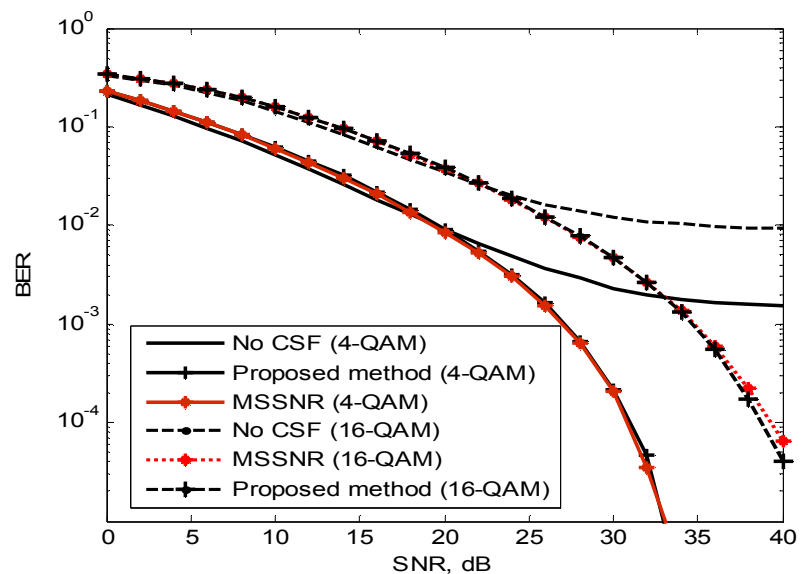

Fig. 6. BER performance versus SNR for $\mathrm{C} 1$
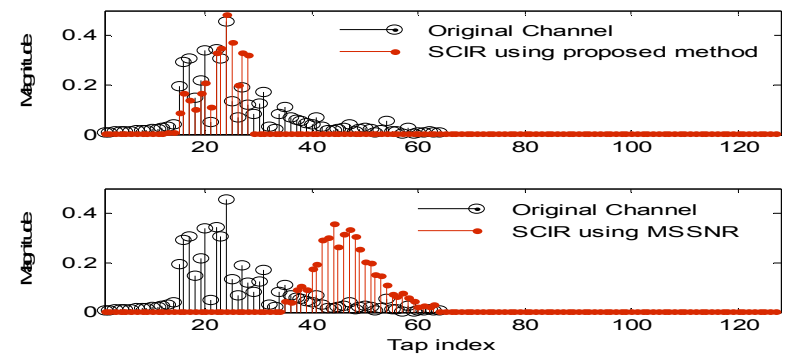

Fig. 7. The original CIR versus the normalized shortened channel C2

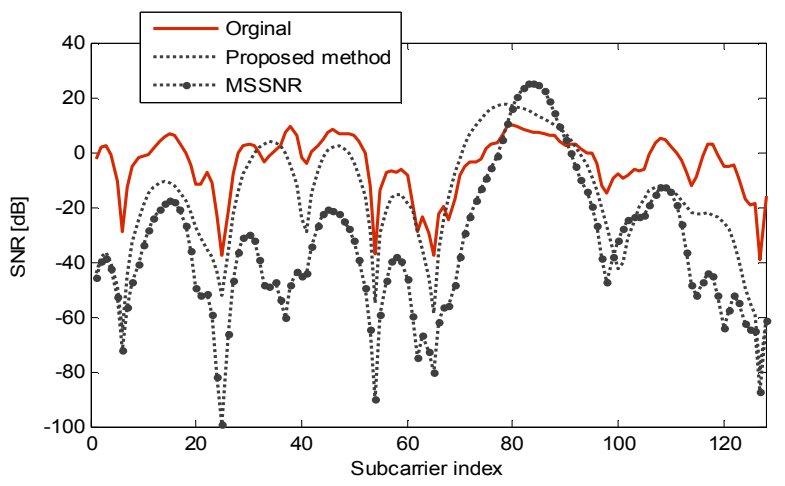

Fig. 8. SNR distribution over frequency for $\mathrm{C} 2$

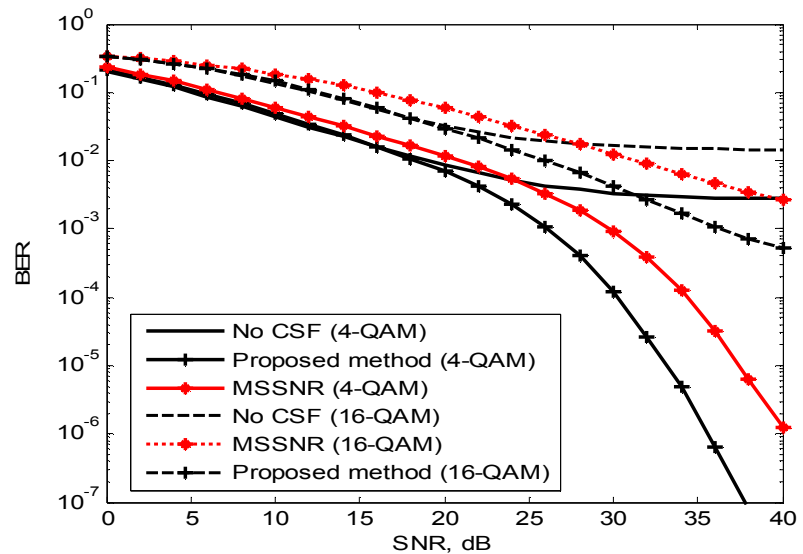

Fig. 9. BER performance versus SNR for $\mathrm{C} 2$ 


\section{CONCLUSION}

A channel shortening design for OFDM systems has been proposed and analyzed. The algorithm is based on energy optimization of the SCIR and on the maximization of the SNR while reducing spectral distortions between the CIR and the SCIR, by exploiting the CSF decomposition [6]. We show by simulations that, the proposed method provides very good BER performance especially in case of channels with long delay spread like CM 4 channel in ultra-wide band communications. Simulation results clearly indicate that the proposed method outperform the MSSNR at the price of a lower computational complexity.

\section{APPENDIX: REVIEW OF THE MSSNR ALGORITHM SYNCHRONIZATION ISSUE [5]}

Let $d$ be the optimal delay introduced by the algorithm to get the best CSF. The MSSNR technique divides the SCIR $\tilde{\boldsymbol{h}}_{d}$ into:

- a desired window of size $L_{\max }+1$, defined as follows

$$
\tilde{\boldsymbol{h}}_{\max }=\left[\tilde{h}_{d}, \tilde{h}_{d+1}, \cdots \tilde{h}_{d+L_{\max }}\right]^{T}
$$

- $\quad$ and a residual window of size $L_{h}+L_{w}-L_{\max }-2$

$$
\tilde{\boldsymbol{h}}_{\min _{d}}=\left[\tilde{h}_{0} \cdots \tilde{h}_{d-1}, \tilde{h}_{d+L_{\max }+1} \cdots \tilde{h}_{L_{h}+L_{w}-2}\right]^{T}
$$

Vectors $\tilde{\boldsymbol{h}}_{\max _{d}}$ and $\tilde{\boldsymbol{h}}_{\text {min }_{d}}$ can be rewritten as follows, after decomposing the convolution matrix into $\boldsymbol{H}_{\text {max }_{d}}$ and $\boldsymbol{H}_{\text {min }_{d}}$

$$
\begin{gathered}
\tilde{\boldsymbol{h}}_{\text {max }_{d}}=\boldsymbol{H}_{\text {max }_{d}} \boldsymbol{w} \\
\text { where } \boldsymbol{H}_{\text {max }_{d}}=\left[\begin{array}{cccc}
h_{d} & h_{d-1} & \cdots & h_{d-L_{w}+1} \\
h_{d+1} & h_{d} & \cdots & h_{d-L_{w}+2} \\
\vdots & \vdots & & \vdots \\
h_{d+L_{\max }} & h_{d+L_{\max }-1} & \cdots & h_{d+L_{\max }-L_{w}+1}
\end{array}\right]
\end{gathered}
$$

and

$$
\tilde{\boldsymbol{h}}_{\text {min }_{d}}=\boldsymbol{H}_{\text {min }_{d}} \boldsymbol{w}
$$

where $\boldsymbol{H}_{\min _{d}}=\left[\begin{array}{llll}h_{0} & 0 & \cdots & 0 \\ \vdots & \ddots & & \vdots \\ h_{d-1} & h_{d-2} & \cdots & h_{d-L_{w}} \\ h_{d+L_{\max }+1} & h_{d+L_{\max }} & \cdots & h_{d+L_{\max }-L_{w}+2} \\ \vdots & \ddots & & \vdots \\ 0 & \cdots & 0 & h_{L_{h}-1}\end{array}\right]$

Desired and residual energy is given, respectively as

$$
\tilde{\boldsymbol{h}}_{\min _{d}}^{H} \tilde{\boldsymbol{h}}_{\min _{d}}=\boldsymbol{w}^{H} \boldsymbol{H}_{\min _{d}}^{H} \boldsymbol{H}_{\min _{d}} \boldsymbol{w}=\boldsymbol{w}^{H} \boldsymbol{A}_{d} \boldsymbol{w}
$$

$$
\tilde{\boldsymbol{h}}_{\max _{d}}^{H} \tilde{\boldsymbol{h}}_{\max _{d}}=\boldsymbol{w}^{H} \boldsymbol{H}_{\text {max }_{d}}^{H} \boldsymbol{H}_{\text {max }_{d}} \boldsymbol{w}=\boldsymbol{w}^{H} \boldsymbol{B}_{d} \boldsymbol{w}
$$

The MSSNR design can be formulated as a single generalized Rayleigh quotient optimization defined as (26). Then, the CSF is the eigenvector that corresponds to the largest generalized eigenvalue [12]. When a delay search algorithm is included, the final CSF is the eigenvector corresponding to the largest eigenvalue, over all $N_{d}$ delay candidates. This solution provides the best bit error ratio.

$$
\boldsymbol{w}_{d}=\underbrace{\arg \max }_{w}\left\{\frac{\boldsymbol{w}^{H} \boldsymbol{A}_{d} \boldsymbol{w}}{\boldsymbol{w}^{H} \boldsymbol{B}_{d} \boldsymbol{w}}\right\}
$$

\section{ACKNOWLEDGMENT}

This work was supported by "la région Bretagne", France through the project CHASHORT.

\section{REFERENCES}

[1] "Expected performance and attribute criteria approved for IEEE P802.15.3a alt PHY selection criteria," IEEE 802.15.3a official website, http: // www .ieee802.org/15/pub/TG3a.html.

[2] R. Schur and J. Speidel, "An efficient equalization method to minimize delay spread in OFDM/DMT systems," Proc. IEEE Int. Conf. on Comm., vol. 5, pp. 1481-1485, June 2001.

[3] D. Falconer and F.R. Magee, "Adaptive channel memory truncation for maximum likelihood sequence estimation," IEEE Trans. on Communications, vol. 62, pp. 1541-1562, November 1973.

[4] S. Celebi, "Interblock interference (IBI) minimizing time-domain equalizer (TEQ) for OFDM," IEEE Signal Processing Letters, vol. 10 , pp.232-234, August 2003.

[5] P. J. W. Melsa, R. C. Younce, and C. E. Rhors, "Impulse response shortening for discrete multitone transceivers," IEEE Trans. on Communications, vol. 44, pp. 1662-1672, December 1996.

[6] K. Ragoubi, M. Hélard, M. Crussière, "Channel shortening for bit rate maximization in DMT communication systems," VTC Fall 2010, pp.1-5.

[7] J.J. Van de Beek, M. Sandell, P.O. Borjesson: "ML estimation of time and frequency offset in OFDM systems", IEEE Trans. Signal Process., 1997, 45, (7), pp. 1800-1805.

[8] D.H Brandwood, "A complex gradient operator and its application in adaptive array theory," IET PROC., vol. 130, Pts. F and H, N0. 1, pp.11-16, February 1983.

[9] R. K. Martin, K. Vanbleu, M. Ding, G. Ysebaert, M. Milosevic, B. L. Evans, M. Moonen, and C. R. Johnson, Jr., "Implementation complexity and communication performance tradeoffs in discrete multitone modulation equalizers," IEEE trans. Signal Process., vol. 54, No. 8, pp. 3216-3230, Aug. 2006.

[10] Hyun-Myung Kim, Dongsik Kim, Gi-Hong Im and Seongwoo Ahn, "Subband interference suppression in channel shortening for OFDMA downlink systems," IEEE Wireless Communications and Networking Conference (WCNC): PHY, pp. 3726-3731, 2013.

[11] A. A. M. Saleh and R.A. Valenzuela, "A statistical model for indoor multipath propagation," IEEE Journal on Selected Areas of Communications, pp. 128-137, February 1987.

[12] G. H. Golub and C. F. Van Loan, Matrix Computations. Baltimore, MD: Johns Hopkins Univ. Press, 1996. 